PROCEEDINGS OF THE

AMERICAN MATHEMATICAL SOCIETY

Volume 128, Number 3, Pages 637-641

S 0002-9939(99)05534-3

Article electronically published on October 25, 1999

\title{
WHEN IS A RIGHT ORDERABLE GROUP LOCALLY INDICABLE?
}

\author{
PATRIZIA LONGOBARDI, MERCEDE MAJ, AND AKBAR RHEMTULLA
}

(Communicated by Ronald M. Solomon)

\begin{abstract}
If a group $G$ has an ascending series $1=G_{0} \leq G_{1} \leq \cdots \leq G_{\rho}=G$ of subgroups such that for each ordinal $\alpha, G_{\alpha} \triangleleft G$, and $G_{\alpha+1} / G_{\alpha}$ has no nonabelian free subsemigroup, then $G$ is right orderable if and only if it is locally indicable. In particular if $G$ is a radical-by-periodic group, then it is right orderable if and only if it is locally indicable.
\end{abstract}

\section{$\S 1$. INTRODUCTION}

Recall that a group $G$ is said to be locally indicable if every finitely generated non-trivial subgroup of $G$ has an infinite cyclic quotient. Such groups are right orderable, as was shown by R.G. Burns and V.W. Hale in [2]. On the other hand, a right orderable group need not be locally indicable as was shown by G.M. Bergman in [1]. It was shown in [9] that a finite extension of a polycyclic group is right orderable only if it is locally indicable. This result was extended to groups which are finite extensions of solvable groups by I.M. Chiswell and P. Kropholler in [3] and to the class of periodic extensions of radical groups by V. M. Tararin in [10]. The purpose of this paper is to show that if a group $G$ has a normal ascending series $1=G_{0} \leq G_{1} \leq \cdots \leq G_{\rho}=G$ of subgroups such that for each ordinal $\alpha<\rho, \quad G_{\alpha+1} / G_{\alpha}$ has no non-abelian free subsemigroup, then $G$ is right orderable only if it is locally indicable.

A group $G$ is said to have a normal ascending series with factors in a class $\mathfrak{X}$ if there is a series $1=G_{0} \leq G_{1} \leq \cdots \leq G_{\rho}=G$ of subgroups such that for each ordinal $\alpha<\rho, G_{\alpha} \triangleleft G$, and $G_{\alpha+1} / G_{\alpha} \in \mathfrak{X}$. $\rho$ is any ordinal number and where $\alpha$ is a limit ordinal, $G_{\alpha}$ is defined to be $\bigcup_{\lambda<\alpha} G_{\lambda}$.

If $N$ is a nilpotent group and $u_{0}, v_{0} \in N$, write $u_{i+1}=u_{i} v_{i}, v_{i+1}=v_{i} u_{i}$ for all $i \geq 0$. Then $u_{r}=v_{r}$ where $r$ is the nilpotency class of $N$. This is a well known result shown by B.H. Neumann and T. Taylor and also by A.I. Mal'cev. Such a group, in particular, contains no non-abelian free subsemigroup. It follows that a locally nilpotent group has no non-abelian free subsemigroups. We shall call a group $G$ an $N F S$-group if it has no non-abelian free subsemigroups. Observe that periodic extensions of $N F S$-groups are again $N F S$-groups.

A group $G$ is called radical if it has a normal ascending series in which all the factors are locally nilpotent groups. We shall call $G$ an NFS-radical group if it has a

Received by the editors March 15, 1998.

1991 Mathematics Subject Classification. Primary 20F19; Secondary 06F15, 20F60.

The third author wishes to thank NSERC for partial financial support.

(C)1999 American Mathematical Society 
normal ascending series in which all the factors are $N F S$-groups. Thus in particular periodic extensions of radical groups are NFS-radical groups. V.M. Tararin [10] has shown that a right orderable radical-by-periodic group is locally indicable. We shall show that a right orderable $N F S$-radical group is locally indicable. We point out that in general, NFS-groups are not periodic extensions of locally nilpotent groups as was shown by A. Yu. Ol'shanski and A. Storozhev in [8].

We wish to thank the referee for his useful comments and have modified results in section 2 as suggested by the referee.

\section{§2. Preliminary material}

Results in this section are not new, but are included to make this paper more or less self-contained.

Lemma 1. Let $G$ be a right orderable group, $\Lambda$ a totally ordered set on which $G$ acts by order preserving permutations, and $G_{\alpha}$ the subgroup of $G$ fixing some element $\alpha \in \Lambda$. Then there is a right order on $G$ with respect to which the subgroup $G_{\alpha}$ is convex.

Proof. Let $\leq_{1}$ be any right order on $G$, and let $\leq_{0}$ be the total order on $\Lambda$. Define relation $\leq$ on $G$ by the rule: $g \leq h \Longleftrightarrow \alpha g \leq_{0} \alpha h$, or $\alpha g=\alpha h$ and $g \leq_{1} h$. It is easy to verify that $(G, \leq)$ is a right ordered group and $G_{\alpha}$ is convex.

If $A$ is a subset of a right ordered group $(G, \leq)$, then $A$ is said to be bounded above (below) if there is some element $g \in G$ such that $a<g(g<a)$ for every $a \in A$. The set $A$ is called unbounded if it is not bounded above or below.

Recall that a subgroup $H$ of a right orderable group $G$ is called relatively convex if it is convex under some right order on $G$. Since the intersection of all relatively convex subgroups of $G$ containing a given set $A$ is relatively convex (see Proposition 5.1 .10 of [6]), there is a unique minimal relatively convex subgroup of $G$ containing $A$. We call this subgroup the relative convex subgroup closure of $A$ in $G$.

Lemma 2. Let $(G, \leq)$ be a right ordered group and let $A$ be a subgroup of $G$. If the set $A$ is bounded above (below), then the relative convex subgroup closure of $A$ in $G$ is a proper subgroup of $G$.

Proof. We prove the lemma in the case $A$ is bounded above. The proof in the other case is similar. Let $\alpha=\{g \in G ; g \leq a$ for some $a \in A\}$. Then $\alpha$ is a convex set and so is $\alpha g$ for any $g \in G$. Note that if $h \notin \alpha g$, then $\alpha h \supset \alpha g$. Order the set $\Lambda=\{\alpha g ; g \in G\}$ by the rule $\alpha h>\alpha g$ if $\alpha h \supset \alpha g$. Then $G$ acts on $\Lambda$ under right multiplication preserving the order on $\Lambda$. Note that $\alpha a=\alpha$ for all $a \in A$ so that $G_{\alpha} \geq A$. By Lemma 1 there is a right order on $G$ under which $G_{\alpha}$ is convex.

Lemma 3. Let $\geq$ be a right order on $G$, and suppose for some element $a>e$ the subgroup $A=\langle a\rangle$ is unbounded in $G$ under $\geq$. Then $g^{-1} a g \geq e$, and $\left\langle g^{-1} a g\right\rangle$ is unbounded for every $g \in G$.

Proof. Take any $h, g \in G$. There exist positive integers $m, n$ such that $g^{-1} \geq a^{-m}$ and $a^{n} \geq h g^{-1}$. Then $g^{-1} a^{m} \geq e$ and $a^{n} g h^{-1} \geq e$. Hence

$$
g^{-1} a^{m+n} g h^{-1} \geq e \quad \text { and } \quad g^{-1} a^{m+n} g \geq h .
$$

Similarly, $\exists r, s \in \mathbb{N}$ such that $a^{r} \geq g^{-1}$ and $h g^{-1} \geq a^{-s}$. Hence

$$
e \geq g^{-1} a^{-r-s} g h^{-1} \text { and } h \geq g^{-1} a^{-r-s} g \text {. }
$$


Take $h=e$ in the first part of the proof to get $g^{-1} a g \geq e$ for every $g \in G$.

Lemma 4. Let $A$ be a normal subgroup of a right ordered group $G$. If $A$ is bounded above (below), then under some right order on $G, A$ is contained in a proper convex normal subgroup of $G$.

Proof. As in the proof of Lemma 2, let $\alpha=\{g \in G ; g \leq a$ for some $a \in A\}$, and $\Lambda=\{\alpha g ; g \in G\}$. Then $G$ acts on $\Lambda$, and $A$ lies in the kernel $K$ of this map since $\alpha g a=\alpha\left(g a g^{-1}\right) g=\alpha g$ for all $g \in G, a \in A$.

That a group of order preserving permutations of an ordered set is right orderable is well known and goes back to papers of Cohn [4], Conrad [5] and Zaitseva [11]. Thus $G / K$ is right orderable.

Take any right order on $G / K$, any right order on $K$, and order $G$ lexicographically by putting every element of $K g$ positive if and only if $K g$ is positive in $G / K$. This is a right order on $G$ with respect to which $K$ is convex.

The statement of Lemma 4 can be made more precise. If $A$ is a normal set, then the relatively convex subgroup closure $\bar{A}$ of $A$ in $G$ is also a normal subgroup. This follows from the two remarks:

1. $\bar{A}$ is the unique minimal relatively convex subgroup of $G$ containing $A$.

2. For any right order on $G$ given by its positive cone $P$, there is a right order on $G$ with positive cone $g^{-1} P g$ for every $g \in G$.

\section{§3. Statement and PRoof of the main Result}

Theorem. A right-orderable NFS-radical group is locally indicable.

Proof of the Theorem. Note that the class of NFS-radical groups is subgroup and quotient closed. Let $G$ be a non-trivial right-orderable $N F S$-radical group. We need to show that every non-trivial finitely generated subgroup of $G$ has a nontrivial torsion-free abelian quotient. To this end we may assume that $G$ is finitely generated. We may further assume that $G$ has no proper non-trivial normal relatively convex subgroup. This assumption is justified because the join of a nested set of relatively convex subgroups is relatively convex as was shown by V.M. Tararin (see Proposition 5.1 .7 of [6]), so that there is a maximal normal relatively convex subgroup of $G$ since $G$ is finitely generated. Thus replace $G$ by a quotient of $G$ with respect to a proper maximal normal relatively convex subgroup, if necessary, so that it has no proper non-trivial normal relatively convex subgroup.

Now $G$ has an ascending series of normal subgroups of $G$ where each factor is an $N F S$-group. Let $A$ be the largest normal $N F S$-subgroup of $G$. Such a subgroup exists since the union of a chain of $N F S$-subgroups of $G$ is again an $N F S$-subgroup. If $A=G$, then $G$ is an NFS-group and by Lemma 9 of [7], every right order on $G$ is a lexicographic right order - by this we mean that the set of convex subgroups form a system where the factor group for every convex jump is order-isomorphic to a subgroup of the additive group of reals. Since $G$ is finitely generated, there is a last convex jump $G_{1} \rightarrow G$, and $G / G_{1}$ is a finitely generated non-trivial torsionfree abelian group. Since $G$ has no proper non-trivial normal relatively convex subgroup, $G_{1}=\langle e\rangle$ and $G$ is abelian. Thus assume $A \neq G$.

Let $\mathfrak{A}=\left\{A_{\alpha} \leq G ; A_{\alpha}=A \cap G_{\alpha}\right.$, where $G_{\alpha}$ is a proper relatively convex subgroup of $G\}$.

For every $A_{\alpha}$ in $\mathfrak{A}$ let $K_{\alpha}$ be the relative convex subgroup closure of $A_{\alpha}$ in $G$. Note that $A_{\alpha}=A \cap K_{\alpha}$. Consider the set $\mathfrak{G}=\left\{K_{\alpha}\right\}$. 
We show that $\mathfrak{G}$, with the inclusion, is an inductive set. Let $\left(K_{i}\right)_{i \in I}$ be a chain in $\mathfrak{G}$. Then the union $\bigcup_{i \in I} K_{i}=L$ is a relatively convex subgroup of $G$. Moreover $L \neq G$, since $G$ is finitely generated. Finally $L \leq T$ for every proper relatively convex subgroup $T$ such that $A \cap T=A \cap L$, because, from $A \cap K_{i} \leq A \cap\left(K_{i} \cap T\right)$ we get $A \cap K_{i}=A \cap K_{i} \cap T$ and $K_{i} \leq K_{i} \cap T$, since $K_{i}$ is minimal. Hence $K_{i} \leq T$ for every $i$, and $L \leq T$.

Therefore $\mathfrak{G}$ is inductive and there exists $G_{0}$ maximal in $\mathfrak{G}$. Let $\leq$ be a right order in $G$ such that $G_{0}$ is convex, and let $\leq_{A}$ be its restriction to $A$. We show that $A_{0}=A \cap G_{0}$ is a maximal convex subgroup of $A$ under $\leq_{A}$. In fact assume there exists $B<A, B$ convex and $A_{0}<B$. Then $B$ is bounded above and, by Lemma 2 , the relatively convex subgroup closure $G_{1}$ of $B$ in $G$ is a proper subgroup of $G$.

Therefore $G_{1}$ is in $\mathfrak{G}$. But we have $A_{0}<B \leq A \cap G_{1}$; hence $A_{0}=A \cap G_{0}=$ $A \cap\left(G_{1} \cap G_{0}\right)$. Since $G_{0} \cap G_{1}$ is relatively convex and $G_{0}$ is the relative subgroup closure of $A_{0}, G_{0} \cap G_{1} \geq G_{0}$. Thus $G_{0}<G_{1}$, a contradiction.

Hence $A_{0}$ is a maximal convex subgroup of $A$. By Lemma 9 of [7], every order on $A$ is lexicographic so that $A_{0}$ is normal in $A$ and $A / A_{0}$ is isomorphic to a subgroup of the additive group of real numbers under the order induced by $\leq$.

It follows that for every positive $a \in A \backslash A_{0}$, the set $\left\{a^{n} \mid n \in \mathbb{Z}\right\}$ is unbounded in $A$; and since $A$ is unbounded in $G$ under every right order on $G$, this set is unbounded in $G$. From Lemma 3 we know that for every $g \in G, g^{-1} a g>e$ and $\left\langle g^{-1} a g\right\rangle$ is unbounded. Thus the set $A \backslash A_{0}$ is invariant and therefore so is the set $A_{0}$.

Now by Lemma 4 and the fact that $G$ has no proper non-trivial normal relatively convex subgroups, it follows that $A_{0}=1$, and the order $\leq_{A}$ is Archimedean. Since $A$ is unbounded in $G$, it follows from Lemma 3 that under conjugation, the action of $G$ on $A$ preserves the order on $A$. Let $K$ be the kernel of this action. Then $G / K$ acts as a group of order preserving automorphisms of the Archimedean ordered group $A$. Thus $G / K$ is isomorphic to a subgroup of the multiplicative group of positive reals which is torsion-free abelian. Hence $G$ has a torsion-free abelian quotient unless $G=K$ and in this case $G$ centralizes $A$.

But $G / A$ is an NFS-radical group, so it has a non-trivial normal subgroup $B / A$ that has no free subsemigroups. We now show that $B$ is an $N F S$-group. For any $x, y \in B$, there are distinct semigroup words $u=u(x, y)$ and $v=v(x, y)$ such that $u=v a$ for some $a \in A$. Then $u v=v u$ since $A$ is in the center of $G$. Thus $B$ has no free subsemigroup, and by our choice of $A, B=A$. Thus $G=A$, contradicting the assumption on $A$.

\section{REFERENCES}

[1] G.M.Bergman, Right-orderable groups that are not locally indicable, Pac. J. Math. 147 (1991), 243-248. MR 92e:20030

[2] R.G.Burns and V.W.Hale, A note on group rings of certain torsion-free groups, Can. Math. Bull. 15 (1972), 441-445. MR 46:9149

[3] I.M.Chiswell and P.H.Kropholler, Soluble right orderable groups are locally indicable, Can. Math. Bull. 36 (1993), 22-29. MR 93j:20088

[4] P.M. Cohn, Groups of order automorphisms of ordered sets, Mathematika 4 (1957), 41-50. MR 19:940e

[5] P.F. Conrad, Right-ordered groups, Mich. Math. J. 6 (1959), 267-275. MR 21:5684

[6] V.M. Kopitov and N. Ya. Medvedev, Right Ordered Groups, Siberian School of Algebra and Logic, Plenum Publishing Corporation, New York, 1996.

[7] P. Longobardi, M. Maj and A. H. Rhemtulla, Groups with no free subsemigroups, Trans. Amer. Math. Soc. 347 (1995), 1419-1427. MR 95g:20043 
[8] A. Yu. Ol'shanski and A. Storozhev, A group variety defined by a semigroup law, J. Austral. Math. Soc. (Series A) 60 (1996), 255-259. MR 97b:20033

[9] A. H. Rhemtulla, Polycyclic right ordered groups, Algebra, Carbondale 1980, Lecture Notes in Mathematics 848 (R.K.Amayo, ed.), Springer Berlin, 1981, pp. 230-234. MR 82i:06025

[10] V.M. Tararin, On Radically right-ordered groups, Sib. Mat. Zh. 32 (1991), 203-204.

[11] M.I. Zaitseva, Right-ordered groups, Uchen. Zap. Shuisk. Gos. Ped. Inst. 6 (1958), 215-226.

Dipartimento di Matematica e Applicazioni "R. Caccioppoli", via Cintia, Monte S. Angelo, 80126, Napoli, Italy

E-mail address: longobar@matna2.dma.unina.it

Dipartimento di Matematica e Informatica, via Salvator Allende, 84081 Baronissi (SALERno), ItALy

E-mail address: maj@matna2.dma.unina.it

Department of Mathematical Sciences, University of Alberta, Edmonton, Alberta, Canada T6G 2G1

E-mail address: akbar@malindi.math.ualberta.ca 